\title{
An Improved INS/VNS Integrated Navigation Measurement Model for Lunar Rover
}

\author{
Yongzhi Xu \\ School of Instrument Science \& Opto-electronics \\ engineering, Beihang University \\ Beijing, China \\ e-mail: buaaxyz@yeah.net
}

\author{
Xiaolin Ning \\ School of Instrument Science \& Opto-electronics \\ engineering, Beihang University \\ Beijing, China \\ e-mail: ningxiaolin@buaa.edu.cn
}

\begin{abstract}
The INS/VNS integrated navigation is an important autonomous navigation method for lunar rovers. The INS/VNS models usually utilize the relative motion differences between the inertial and the vision as measurements. Traditional INS/VNS models make an approximation between definitions of the inertial measured attitude error and the misalignment angle to simplify the measurement model of relative rotation difference. This paper finds that the norm of the approximation error can exceed the norm of misalignment angle when the yaw is large, which cannot be ignored. Furthermore, an improved measurement model without that approximation is presented, in which the definition of inertial measured attitude error and the misalignment angle is accurately matched. Lunarbased simulation and earth-based experiment both demonstrate that the presented model is effective and the method used the proposed model can achieve higher accuracy of positions and attitudes than that used the traditional model.
\end{abstract}

Keywords-Inertial navigation; Vision navigation; Integrated navigation; Measurement model; Lunar rover

\section{INTRODUCTION}

High-accuracy autonomous navigation is one of the key technologies for planetary rovers. Inertial and vision integrated navigation (INS/VNS) methods are usually used for planetary rovers, such as in China's first lunar rover, Yutu [1], and NASA's Curiosity [2]. INS/VNS methods $[3,4]$ usually utilize the inertial information to build the state model, and the vision information to build the measurement model.

Traditional INS/VNS models make an approximation between the definition of inertial measured attitude error and misalignment angle to simplify the measurement model $[5,6,7]$. However, the norm of the approximation error can exceed the norm of the misalignment angle when the yaw is large, which is always ignored in the traditional models. A new measurement model is presented to avoid the approximation. Lunar-based simulation and earth based experiment both demonstrate that the position and attitude accuracies achieved by the method with the proposed model have improved significantly compared with one used the traditional model.

\section{REFERENCE FRAMES}

The reference coordinate frames are all defined as right hand frames, including the inertial frame $\left(i, o_{m} x_{i} y_{i} z_{i}\right)$, the moon fixed frame $\left(m, o_{m} x_{m} y_{m} z_{m}\right)$, the geographic frame $\left(t, o_{b} x_{t} y_{t} z_{t}\right)$, the world frame $\left(w, o_{w} x_{w} y_{w} z_{w}\right)$, the body frame $\left(b, o_{b} x_{b} y_{b} z_{b}\right)$ and the camera frame $\left(c, o_{c} x_{c} y_{c} z_{c}\right)$, some of which are show in Fig .1. In $t$-frame, the $\mathrm{x}$-axis points to the east and the $\mathrm{y}$-axis points to the north. The $w$ frame is defined as the first instant $t$-frame. In $b$-frame, the $\mathrm{x}$-axis points to the right of the rover and the $\mathrm{y}$-axis points to the front of the rover. The origin of $c$-frame is at the optical center of left camera. In $c$-frame, $x$-axis points to the right of the camera and $y$-axis points downward.

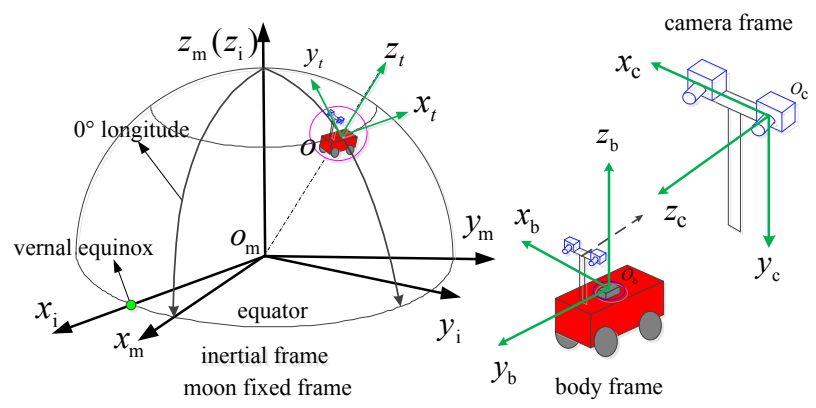

Figure 1 Coordinate frames

\section{STATE MODEL}

The state $\boldsymbol{X}$ contains the motion errors and the IMU drifts:

$$
\boldsymbol{X}=\left[\begin{array}{lllll}
\boldsymbol{\phi}^{T} & \delta \boldsymbol{v}^{T} & \delta \boldsymbol{r}^{T} & \boldsymbol{\varepsilon}^{T} & \nabla^{T}
\end{array}\right]^{T}
$$

where $\phi$ is the attitude error angle, $\delta \boldsymbol{v}$ is the velocity error, $\boldsymbol{\delta} \boldsymbol{r}$ is the position error, $\boldsymbol{\varepsilon}$ is the gyroscopes drift, and $\nabla$ is the accelerometers bias.

The state model is built based on the inertial error equation [8]: 


$$
\left\{\begin{array}{l}
\phi^{\&}=-\omega_{i w}^{w} \times \phi+R_{\mathrm{b}}^{\mathrm{w}} \boldsymbol{\varepsilon} \\
\delta \&=-\left(\boldsymbol{R}_{\mathrm{b}}^{\mathrm{w}} \boldsymbol{f}^{\mathrm{b}}\right) \times \phi-2 \omega_{i w}^{w} \times \delta \boldsymbol{v}+\boldsymbol{R}_{\mathrm{b}}^{\mathrm{w}} \nabla \\
\delta \&=\delta \boldsymbol{v} \\
\&=0 \\
\&=\mathbf{0}
\end{array}\right.
$$

where $\omega_{i b}^{b}$ is the rotation velocity measured by gyroscopes, $\boldsymbol{f}^{b}$ is the acceleration measured by accelerometers. $\boldsymbol{R}_{\mathrm{w}}^{\mathrm{b}}$ is the attitude matrix, $\boldsymbol{\omega}_{i w}^{w}$ is the angular velocity of the $w$ frame relative to the $i$-frame expressed in the $w$-frame.

\section{Measurement Model Based on RELATIVE MOTION DIFFERENCE}

\section{A. Measurement of The Relative Motion Difference}

The frequency of the vision data is commonly lower than that of the inertial data, so it is also used as the data fusion frequency. All the $t_{k}$ in this paper denotes the $\mathrm{k}$ instant of data fusion process.

$b_{k}$ denotes the $b$-frame at $t_{k} \cdot \boldsymbol{q}_{b_{k-1}}^{b_{k}}$ and $\boldsymbol{T}_{b_{k-1} b_{k}}^{b_{k-1}}$ are the relative rotation quaternion and translation vector from $b_{k}$ to $b_{k-1}$, which are usually called as relative motion. The difference of the inertial and the vision relative motion is used as the measurement.

1) Inertial relative motion: $\boldsymbol{q}_{b_{k-1} b_{k}, \text { INS }}^{b_{k-1}}$ and $\boldsymbol{T}_{b_{k-1} b_{k}, \text { INS }}^{b_{k-1}}$ are the inertial relative motion, which can be computed as:

$$
\begin{aligned}
& \boldsymbol{q}_{b_{k-1}, \mathrm{INS}}^{b_{k}}=\hat{\boldsymbol{q}}_{b_{k-1}}^{w} \mathrm{o} \boldsymbol{q}_{w, \mathrm{INS}}^{b_{k}} \\
& \boldsymbol{T}_{b_{k-1} b_{k}, \mathrm{INS}}^{b_{k-1}}=\hat{\boldsymbol{R}}_{w}^{b_{k-1}}\left(\boldsymbol{r}_{k, \mathrm{INS}}-\hat{\boldsymbol{r}}_{k-1}\right)
\end{aligned}
$$

where $\hat{\boldsymbol{R}}_{w}^{b_{k-1}}$ and $\hat{\boldsymbol{r}}_{k-1}$ are INS/VNS estimated attitude matrix and position vector at $t_{\mathrm{k}-1}$ respectively. $\hat{\boldsymbol{q}}_{b_{k-1}}^{w}$ is the quaternion of $\hat{\boldsymbol{R}}_{w}^{b_{k-1}} \cdot \boldsymbol{q}_{w, \text { INS }}^{b_{k}}$ and $\boldsymbol{r}_{k, \mathrm{INS}}$ are inertial calculated attitude quaternion and position at $t_{\mathrm{k}}$ respectively.

2) Vision relative motion: The procedure of VNS mainly includes feature extraction, feature tracking, stereo matching, 3D reconstruction and motion estimation $[9,10,11]$. By $3 \mathrm{D}$ reconstruction, the coordinates of feature points in c-frame are $\boldsymbol{r}_{c, i}(k)(i=1 \mathrm{~L} \mathrm{~N})$, where $\mathrm{N}$ is the number of feature points. $\boldsymbol{R}_{c_{k-1}, \mathrm{VNS}}^{c_{k}}$ and $\boldsymbol{T}_{c_{k-1} c_{k}, \mathrm{VNS}}^{c_{k-1}}$ are the vision relative motion parameters in $c$-frame, which can be calculated as follows:

$$
\boldsymbol{r}_{c, i}(k)=\boldsymbol{R}_{c_{k-1}, V N S}^{c_{k}}\left[\boldsymbol{r}_{c, i}(k-1)+\boldsymbol{T}_{c_{k-1} c_{k}, \mathrm{VNS}}^{c_{k-1}}\right](i=1 \mathrm{~L} N)
$$

$\boldsymbol{R}_{b_{k-1}, \text { VNS }}^{b_{k}}$ and $\boldsymbol{T}_{b_{k-1} b_{k}, \text { VNS }}^{b_{k-1}}$ are the corresponding vision relative motion parameters in $b$-frame, which can be calculated as follows:

$$
\begin{aligned}
& \boldsymbol{R}_{b_{k-1}, \mathrm{VNS}}^{b_{k}}=\boldsymbol{R}_{c}^{b} \boldsymbol{R}_{c_{k-1}, \mathrm{VNS}}^{c_{k}} \boldsymbol{R}_{b}^{c} \\
& \boldsymbol{T}_{b_{k-1} b_{k}, \mathrm{VNS}}^{b_{k}}=\boldsymbol{R}_{c}^{b} \boldsymbol{T}_{c_{k-1} c_{k}, \mathrm{VNS}}^{c_{k-1}}+\boldsymbol{R}_{c}^{b}\left(\boldsymbol{R}_{c_{k}, \mathrm{VNS}}^{c_{k-1}}-\boldsymbol{I}\right) \boldsymbol{T}_{c}^{b}
\end{aligned}
$$

where $\boldsymbol{R}_{c}^{b}$ and $\boldsymbol{T}_{c}^{b}$ are the rotation matrix and translation vector from $c$-frame to $b$-frame.
The measurement can be computed as:

$$
\boldsymbol{z}=\left[\begin{array}{c}
\boldsymbol{z}_{q} \\
\boldsymbol{z}_{r}
\end{array}\right]=\left[\begin{array}{c}
\boldsymbol{f}_{q}\left(\boldsymbol{q}_{b_{k-1}, \mathrm{VNS}}^{b_{b_{k}}}{ }^{-1} \mathrm{oq}_{b_{k-1}, \mathrm{INS}}^{b_{k}}\right) \\
\boldsymbol{T}_{b_{k-1} b_{k}, \mathrm{INS}}^{b_{\mathrm{N}}}-\boldsymbol{T}_{b_{k-1} b_{k}, \mathrm{VNS}}^{b_{k-1}}
\end{array}\right]
$$

where $\boldsymbol{f}_{q}(\boldsymbol{q})=\lambda^{T}$ when $\boldsymbol{q}=\left[\begin{array}{ll}\lambda_{0} & \lambda\end{array}\right]^{T}$.

\section{B. Traditional Measurement model}

To simplify the measurement model, the traditional methods analyze the error of the relative motion as follows.

1) Traditional measurement model of $\boldsymbol{z}_{q}$ : To simplify the measurement model, the traditional methods define the error of $\boldsymbol{q}_{w, \mathrm{INS}}^{b}$ as:

$$
\delta \boldsymbol{q}_{\mathrm{INS}}=\boldsymbol{q}_{b}^{w} \mathrm{o} \boldsymbol{q}_{w, \mathrm{INS}}^{b}
$$

where $\boldsymbol{q}_{w}^{b}$ is the real attitude quaternion.

As the definition of $\phi$ is:

$$
\boldsymbol{I}-[\boldsymbol{\phi} \times]=\boldsymbol{R}_{b}^{w} \boldsymbol{R}_{w, \mathrm{INS}}^{b}
$$

the relationship of $\boldsymbol{\delta} \boldsymbol{q}_{\mathrm{INS}}$ and $\boldsymbol{\phi}$ is:

$$
\delta \boldsymbol{q}_{\mathrm{INS}}=\boldsymbol{q}_{b}^{w} \mathrm{\circ}\left[\begin{array}{c}
1 \\
\frac{1}{2} \boldsymbol{\phi}
\end{array}\right] \mathrm{o} \boldsymbol{q}_{w}^{b} \approx \boldsymbol{q}_{b}^{w} \mathrm{o}\left[\begin{array}{c}
1 \\
\frac{1}{2} \boldsymbol{\phi}
\end{array}\right]
$$

$\boldsymbol{q}_{b_{k-1}, \text { INS }}^{b_{k}}$ can be expressed as:

$$
\begin{aligned}
\boldsymbol{q}_{b_{k-1}, \mathrm{INS}}^{b_{k}} & =\hat{\boldsymbol{q}}_{b_{k-1}}^{w} \mathrm{o} \boldsymbol{q}_{w, \mathrm{INS}}^{b_{k}} \\
& =\boldsymbol{q}_{b_{k-1}}^{w} \mathrm{o} \boldsymbol{q}_{w}^{b_{k}} \mathrm{o} \boldsymbol{\delta} \boldsymbol{q}_{\mathrm{INS}, k} \\
& =\boldsymbol{q}_{b_{k-1}}^{b_{k}} \mathrm{o} \boldsymbol{\delta} \boldsymbol{q}_{\mathrm{INS}, k}
\end{aligned}
$$

The error of $\boldsymbol{q}_{b_{k-1}, \text { VNS }}^{b_{k}}$ is defined as:

$$
\boldsymbol{q}_{b_{k-1}, \mathrm{VNS}}^{b_{k}}=\boldsymbol{q}_{b_{k-1}}^{b_{k}} \mathrm{o} \boldsymbol{\delta} \boldsymbol{q}_{\mathrm{VNS}, k}=\boldsymbol{q}_{b_{k-1}}^{b_{k}} \mathrm{o}\left[\begin{array}{c}
1 \\
\frac{1}{2} \boldsymbol{\phi}_{\mathrm{NNS}, k}
\end{array}\right]
$$

The measurement model of $\boldsymbol{z}_{q}$ can be expressed as:

$$
\boldsymbol{z}_{q}=\boldsymbol{f}_{q}\left(\boldsymbol{q}_{b_{k-1, \mathrm{NNS}}^{b_{k}}-1} \mathrm{oq}_{b_{k-1}, \mathrm{INS}}^{b_{k}}\right)=\frac{1}{2} \boldsymbol{\phi}_{k}-\frac{1}{2} \boldsymbol{\phi}_{\mathrm{NNS}, k}
$$

2) Traditional measurement model of $\boldsymbol{z}_{r}$ : As the position error state $\boldsymbol{\delta} \boldsymbol{r}=\boldsymbol{r}_{\mathrm{INS}}-\boldsymbol{r}, \boldsymbol{T}_{b_{k-1} b_{k}, \mathrm{INS}}^{b_{k-1}}$ and $\boldsymbol{T}_{b_{k-1} b_{k}, \mathrm{VNS}}^{b_{k-1}}$ can be written as:

$$
\begin{aligned}
& \boldsymbol{T}_{b_{k-1} b_{k}, \mathrm{INS}}^{b_{k-1}}=\boldsymbol{T}_{b_{k-1} b_{k}}^{b_{k-1}}+\hat{\boldsymbol{R}}_{w}^{b_{k-1}} \boldsymbol{\delta} \boldsymbol{r}_{k} \\
& \boldsymbol{T}_{b_{k-1} b_{k}, \mathrm{VNS}}^{b_{k-1}}=\boldsymbol{T}_{b_{k-1} b_{k}}^{b_{k-1}}+\Delta \boldsymbol{T}_{\boldsymbol{k}, \mathrm{VNS}}
\end{aligned}
$$

The measurement model of $\boldsymbol{z}_{r}$ can be expressed as:

$$
\boldsymbol{z}_{r}=\boldsymbol{T}_{b_{k-1} b_{k}, \mathrm{NNS}}^{b_{k-1}}-\boldsymbol{T}_{b_{k-1} b_{k}, \mathrm{VNS}}^{b_{k-1}} \approx \hat{\boldsymbol{R}}_{w}^{b_{k-1}} \boldsymbol{\delta} \boldsymbol{r}_{k}+\Delta \boldsymbol{T}_{k, \mathrm{VNS}}
$$

3) Traditional measurement model: In summary, the traditional measurement model can be expressed as:

$$
\boldsymbol{z}(\boldsymbol{k})=\boldsymbol{H}_{1} \boldsymbol{X}(\boldsymbol{k})+\boldsymbol{V}_{1}
$$

$$
\boldsymbol{H}_{1}=\left[\begin{array}{c}
\boldsymbol{H}_{q 1} \\
\boldsymbol{H}_{r 1}
\end{array}\right]=\left[\begin{array}{cccc}
\frac{1}{2} \boldsymbol{I}_{3 \times 3} & \boldsymbol{0}_{3 \times 3} & \boldsymbol{0}_{3 \times 3} & \boldsymbol{0}_{3 \times 6} \\
\boldsymbol{0}_{3 \times 3} & \boldsymbol{0}_{3 \times 3} & \hat{\boldsymbol{R}}_{w}^{b_{k-1}} & \boldsymbol{0}_{3 \times 6}
\end{array}\right]
$$




$$
V_{1}=\left[\begin{array}{c}
-\frac{1}{2} \phi_{\mathrm{NNS}, k} \\
\Delta \boldsymbol{T}_{\boldsymbol{k}, \mathrm{VNS}}
\end{array}\right]
$$

\section{Error Analysis of Traditional Measurement model}

The traditional measurement model makes an approximation between the definition of $\delta \boldsymbol{q}_{\mathrm{INS}}$ and $\phi$ to simplify the measurement model of $\boldsymbol{z}_{q}$ in (9). The error caused by the approximation can be written as $\Delta \boldsymbol{q}$ :

$$
\Delta \boldsymbol{q}=\boldsymbol{q}_{b}^{w} \mathrm{o}\left[\begin{array}{c}
1 \\
\frac{1}{2} \boldsymbol{\phi}
\end{array}\right] \mathrm{o} \boldsymbol{q}_{w}^{b} \mathrm{o}\left[\begin{array}{c}
1 \\
-\frac{1}{2} \boldsymbol{\phi}
\end{array}\right]
$$

The angle of $\Delta \boldsymbol{q}$ is $\Delta \boldsymbol{\phi}$. Define $\Delta P$ to describe the relative error of $\Delta \phi$ :

$$
\Delta P=\frac{|\Delta \phi|}{|\phi|} * 100 \%
$$

Fig .2 shows the $\Delta P$ analysis curve when the yaw is from $0^{\circ}$ to $360^{\circ}$, the pitch and roll is $3^{\circ}$, and $\phi=\left[\begin{array}{lll}1 & 1 & 1\end{array}\right]^{\mathrm{T}} * 0.02^{\circ}$. It can be seen from Fig .2, $\Delta P$ changes from 0 to $160 \%$, and can exceed $100 \%$ when the yaw is more than $110^{\circ}$. Meanwhile, the change of pitch and roll from $-5^{\circ}$ to $5^{\circ}$, and the change of $\phi$ norm do not affect $\Delta P$. In summary, the $\Delta \phi$ norm can exceed $\phi$ norm when the yaw is large, which cannot be ignored.

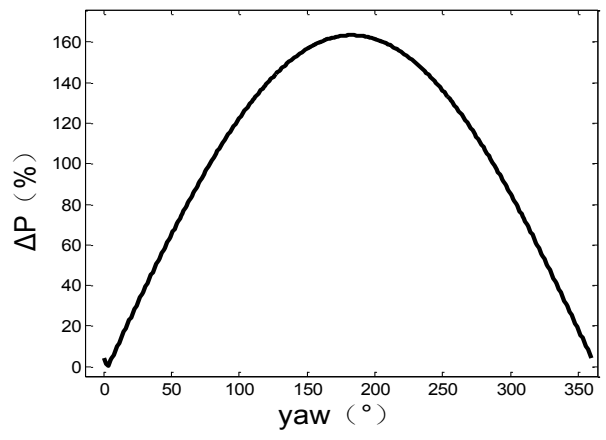

Figure $2 \Delta P$ analysis

\section{New Measurement Model of The Relative Rotation Difference}

To avoid the approximation between the definition of $\delta \boldsymbol{q}_{\mathrm{INS}}$ and $\boldsymbol{\phi}$ in the traditional method, an improved relative rotation difference measurement model is presented. The new error definition of $\boldsymbol{q}_{w, \mathrm{NNS}}^{b}$ is:

$$
\Delta \boldsymbol{q}_{\mathrm{INS}}=\boldsymbol{q}_{w, \mathrm{INS}}^{b} \mathrm{o} \boldsymbol{q}_{b}^{w}
$$

According to the definition of $\phi$ in the state model (2), there is an accurate relationship as follows:

$$
\Delta \boldsymbol{q}_{\mathrm{INS}}=\left[\begin{array}{c}
1 \\
\frac{1}{2} \phi
\end{array}\right]
$$

Then $\boldsymbol{q}_{b_{k-1}, \text { INS }}^{b_{k}}$ can be expressed as:

$$
\begin{aligned}
\boldsymbol{q}_{b_{k-1}, \mathrm{INS}}^{b_{k}}= & \hat{\boldsymbol{q}}_{b_{k-1}}^{w} \mathrm{o} \boldsymbol{q}_{w, \mathrm{INS}}^{b_{k}} \\
= & \boldsymbol{q}_{b_{k-1}}^{w} \mathrm{o} \boldsymbol{q}_{w, \mathrm{INS}}^{b_{k}} \\
= & \boldsymbol{q}_{b_{k-1}}^{b_{k}} \mathrm{o} \boldsymbol{q}_{b_{k}}^{w} \mathrm{o} \Delta \Delta \boldsymbol{q}_{\mathrm{INS}, k} \mathrm{o} \boldsymbol{q}_{w}^{b_{k}} \\
= & \boldsymbol{q}_{b_{k-1}}^{b_{k}} \mathrm{o}\left(\boldsymbol{q}_{b_{k}, \mathrm{INS}}^{w} \mathrm{o} \Delta \boldsymbol{q}_{\mathrm{INS}, k}\right) \mathrm{o} \\
& \Delta \boldsymbol{q}_{\mathrm{INS}, k} \mathrm{o}\left(\Delta \boldsymbol{q}_{\mathrm{INS}, k}^{-1} \mathrm{o} \boldsymbol{q}_{w, \mathrm{INS}}^{b_{k}}\right) \\
= & \boldsymbol{q}_{b_{k-1}}^{b_{k}} \mathrm{o} \boldsymbol{q}_{b_{k}, \mathrm{INS}}^{w} \mathrm{o} \Delta \boldsymbol{q}_{\mathrm{INS}, k} \mathrm{o} \boldsymbol{q}_{w, \mathrm{INS}}^{b_{k}}
\end{aligned}
$$

The corresponding measurement model of $\boldsymbol{z}_{q}$ is:

$$
\begin{aligned}
\boldsymbol{z}_{q}= & \boldsymbol{f}_{q}\left(\boldsymbol{q}_{b_{k-1, \mathrm{NNS}} b_{k}}^{-1} \mathrm{o} \boldsymbol{q}_{b_{k-1}, \mathrm{INS}}^{b_{k}}\right) \\
= & \boldsymbol{f}_{q}\left(\boldsymbol{\delta} \boldsymbol{q}_{\mathrm{VNS}, k}{ }^{-1} \mathrm{o} \boldsymbol{q}_{b_{k}, \mathrm{INS}}^{w} \mathrm{o} \Delta \boldsymbol{q}_{\mathrm{INS}, k} \mathrm{o} \boldsymbol{q}_{w, \mathrm{INS}}^{b_{k}}\right) \\
= & \boldsymbol{f}_{q}\left(\left[\begin{array}{c}
1 \\
-\frac{1}{2} \boldsymbol{\phi}_{\mathrm{NS}, k}
\end{array}\right] \mathrm{o} \boldsymbol{q}_{b_{k}, \mathrm{INS}}^{w} \mathrm{o}\left[\begin{array}{c}
1 \\
\frac{1}{2} \boldsymbol{\phi}_{\mathrm{k}}
\end{array}\right] \mathrm{o} \boldsymbol{q}_{w, \mathrm{INS}}^{b_{k}}\right) \\
= & \boldsymbol{f}_{q}\left(\boldsymbol{M}^{*}\left(\boldsymbol{q}_{w, \mathrm{INS}}^{b_{k}}\right) \boldsymbol{M}\left(\boldsymbol{q}_{b_{k}, \mathrm{NNS}}^{w}\right)\left[\begin{array}{c}
1 \\
\frac{1}{2} \boldsymbol{\phi}_{\mathrm{k}}
\end{array}\right]\right) \\
& +\boldsymbol{f}_{q}\left(\left[\begin{array}{c}
0 \\
-\frac{1}{2} \boldsymbol{\phi}_{\mathrm{NN}, k}
\end{array}\right] \mathrm{o} \boldsymbol{q}_{b_{k}, \mathrm{INS}}^{w} \mathrm{o}\left[\begin{array}{c}
1 \\
\frac{1}{2} \boldsymbol{\phi}_{\mathrm{k}}
\end{array}\right] \mathrm{o} \boldsymbol{q}_{w, \mathrm{NS}}^{b_{k}}\right) \\
= & \boldsymbol{H}_{q 2} \boldsymbol{X}+\boldsymbol{V}_{q 2}
\end{aligned}
$$

where $\boldsymbol{V}_{q 2}$ is the corresponding measurement noise as follows:

$$
\boldsymbol{V}_{q 2}=\boldsymbol{f}_{q}\left(\left[\begin{array}{c}
0 \\
-\frac{1}{2} \boldsymbol{\theta}_{\mathrm{VNS}, k}
\end{array}\right] \mathrm{o} \boldsymbol{q}_{b_{k}, \mathrm{INS}}^{w} \mathrm{o} \delta \boldsymbol{q}_{\mathrm{INS}, k} \mathrm{o} \boldsymbol{q}_{w, \mathrm{INS}}^{b_{k}}\right)
$$

$\boldsymbol{H}_{q 2}$ is the corresponding improved measurement matrix:

$$
\boldsymbol{H}_{q 2}=\left[\frac{1}{2} \boldsymbol{f}_{M}\left(\boldsymbol{M}^{*}\left(\boldsymbol{q}_{w, \mathrm{NSS}}^{b_{k}}\right) \boldsymbol{M}\left(\boldsymbol{q}_{b_{k}, \mathrm{NS}}^{w}\right)\right) \quad \boldsymbol{0}_{3 \times 12}\right]
$$

where the function $\boldsymbol{f}_{\boldsymbol{M}}(\boldsymbol{M})$ is defined as:

$$
\boldsymbol{M}_{4 \times 4}=\left[\begin{array}{ll}
\left(M_{1}\right)_{1 \times 1} & \left(M_{2}\right)_{1 \times 3} \\
\left(M_{3}\right)_{3 \times 1} & \left(M_{4}\right)_{3 \times 3}
\end{array}\right], f_{M}(M)=\left(M_{4}\right)_{3 \times 3}
$$

\section{SIMULATION AND EXPERIMENT}

\section{A. Simulation}

Due to limitations in experimental conditions and cost, real experiments on the Moon cannot be achieved. The Moon-simulated IMU data and images are produced by a simulation system. The IMU data is produced by an inertial path generator and the images are produced by a 3DS MAX scene. The 3DS MAX scene is built based on images of the lunar surface from NASA, as shown in Fig .3 typically. 


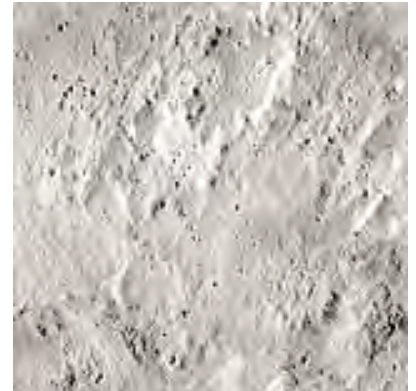

Figure 3 A typical lunar surface image

1) Simulation conditions: The constant bias and random drift of gyroscopes are $1 \% / \mathrm{h}$ and $0.5 \% \mathrm{~h}$, respectively. The constant bias and random drift of the accelerometers are $100 \mu \mathrm{g}$ and $50 \mu \mathrm{g}$, respectively. The simulated binocular visual system is made up of a pair of parallel installed cameras, with the baseline of $20 \mathrm{~cm}$ and the height of $1.7 \mathrm{~m}$ from the surface. The image resolution is $1392 \times 1040$ pixels, and the view field is $45.76^{\circ} \times 35.00^{\circ}$. The frequencies of INS and VNS are $100 \mathrm{HZ}$ and $0.2 \mathrm{HZ}$ respectively.

2) The simulation results: The simulation trajectories of INS, VNS, traditional INS/VNS(INT1) and improved INS/VNS(INT2) navigation methods are shown in Fig .4. As can be seen in Fig .4, the position errors of INT1 and INT2 are quite smaller than that of VNS, which demonstrate that the two integrated navigation methods are effective. Furthermore, the INT2 trajectory is closer to the ideal trajectory than INT1 in the whole trace, which indicates the position accuracy improvement of INT2 over INT1. Fig .5 gives the attitude errors comparison results between INT1 and INT2. As can be seen from Fig .5, the pitch and roll errors of INT2 fluctuate more gently and smaller than INT1. The yaw error of INT2 diverges more slightly than INT1, which can improve the position accuracy significantly [12].

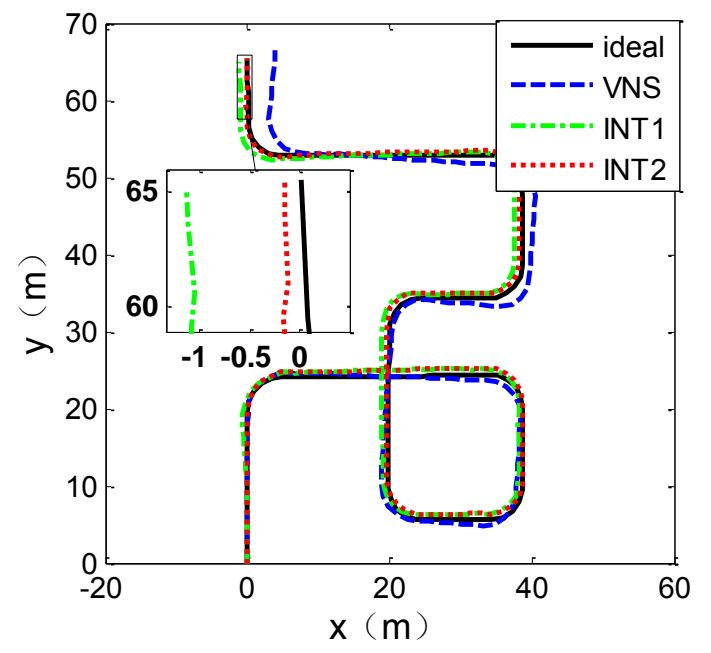

Figure 4 Simulation trajectories

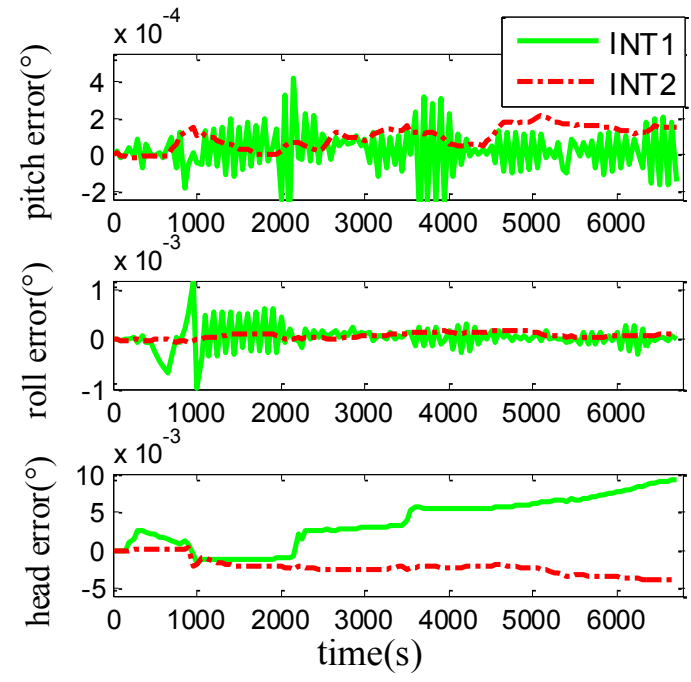

Figure 5 Simulation attitude errors

Table 1 gives the detailed comparison results of final position and attitude errors between four different navigation methods. It can be seen from Table 1, VNS achieves higher position accuracy than INS, while INS achieves higher attitude accuracy than VNS. The position and attitude accuracies of both INT1 and INT2 are higher than INS and VNS, which demonstrates that the two integrated INS/VNS navigation methods are effective. The position error of INT1 is $1.238 \mathrm{~m}$, which is $0.611 \%$ of the trace length $204.24 \mathrm{~m}$. The position error of INT2 is $0.079 \%$ of the trace length, which has been improved by $87.07 \%$ compared with INT1. INT2 achieves higher attitude accuracies than INT1, especially in the yaw direction. The yaw error of INT2 is $-0.221^{\circ}$, which has improved $58.46 \%$ of INT2's $0.532^{\circ}$. As the position accuracy of vision system is sensitive to the yaw accuracy [12], the increase of yaw accuracy in INT2 is extremely beneficial for the position accuracy improvement. In summary, there is a significant improvement in both position and attitude performance of INT2 over INS, VNS and INT1.

TABLE I. SIMULATION FINAL POSITION AND ATTITUDE ERRORS

\begin{tabular}{cccccc}
\hline \multirow{2}{*}{ methods } & \multicolumn{2}{c}{ final position errors } & \multicolumn{3}{c}{ final attitude errors $\left({ }^{\circ}\right)$} \\
\cline { 2 - 6 } & $\begin{array}{c}\text { absolute } \\
\text { value }(\mathrm{m})\end{array}$ & $\begin{array}{c}\text { percentage } \\
(\%)\end{array}$ & pitch & roll & yaw \\
\hline INS & 21386 & 10475 & 0.609 & 0.596 & 1.876 \\
VNS & 4.086 & 2.001 & 1.050 & -0.098 & -6.262 \\
INT1 & 1.238 & 0.611 & -0.008 & 0.000 & 0.532 \\
INT2 & 0.159 & 0.079 & 0.008 & 0.007 & -0.221 \\
\hline
\end{tabular}

B. Experiment

1) Experiment conditions: Though there are differences of the gravity, rotational speed and surface images between on the Earth and on the Moon, the earthbased experiment is feasible and beneficial to verify the navigation methods. The dataset 2011_09_30_drive_0028 of KITTI Vision [13] is used in the experiment, in which the car drives 8.38 minutes and $4128.86 \mathrm{~m}$.

The constant bias of the gyroscopes is $0.01 \%$, the constant bias of accelerometers is $1020.4 \mu \mathrm{g}$. The frequency of IMU data is $100 \mathrm{HZ}$. The baseline of the 
binocular cameras is $54 \mathrm{~cm}$ and the height from the ground surface is $1.65 \mathrm{~m}$. The image resolution is $1226 \times 370$, and the image frequency is $10 \mathrm{HZ}$. A typical image taken in the experiment is shown in Fig .6.

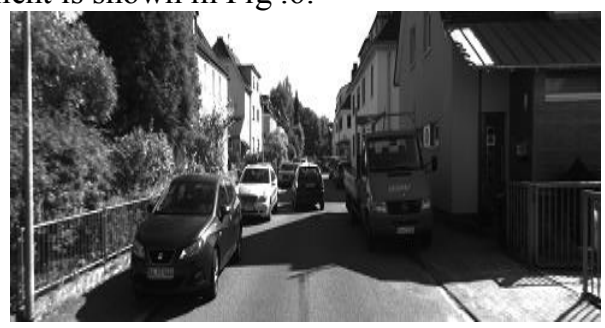

Figure 6 A typical experiment image

2) Experiment results: Fig .7 shows the position trajectories comparison, from which can been clearly seen that INT2 has higher position accuracy than INT1 and VNS. Fig .8 the experimental attitude errors of INT1 and INT2, which shows that the pitch and roll errors of INT2 is a bit smoother and smaller than INT1. And the yaw error of INT2 is much smmother than that of INT1, which diverges slightly.

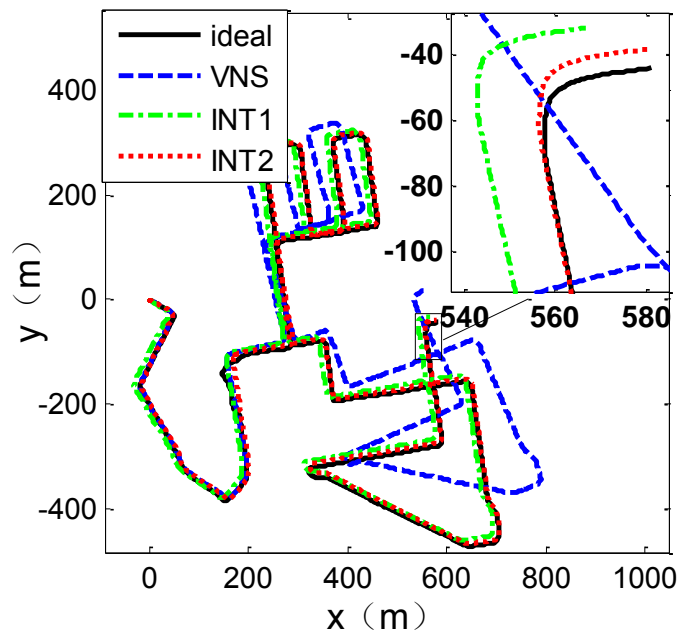

Figure 7 Experiment trajectories

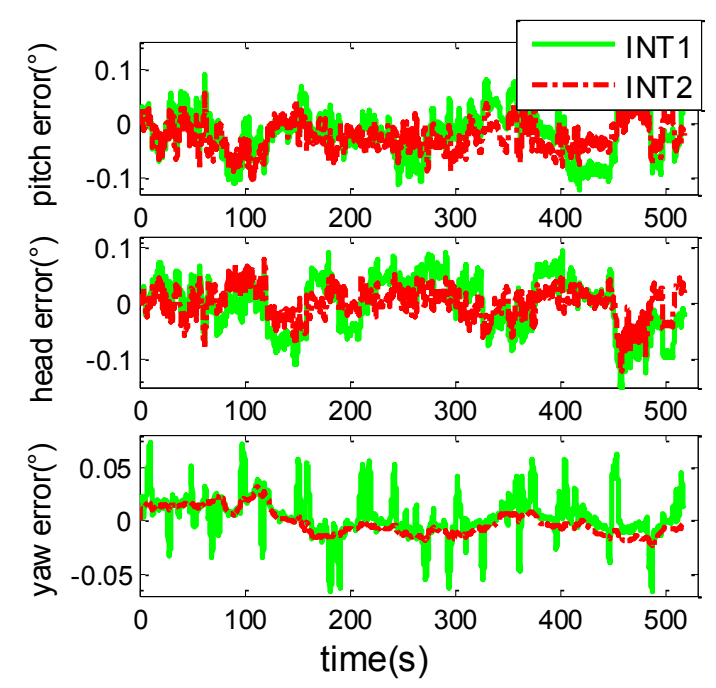

Figure 8 Experiment attitude errors
Table 2 shows the final position and attitude errors of the four navigation methods. It can be seen from Table 2, the position accuracy of INT2 is $0.143 \%$, which has been improved by $68.64 \%$ compared with INT1's $0.456 \%$. The attitude accuracies of INT2 are all higher than that of INT1. Especially, the yaw accuracy of INT2 is $-0.105^{\circ}$, which has been improved by $87.04 \%$ compared with INT1's $0.810^{\circ}$.

Table 2. Experiment final position and attitude errors

\begin{tabular}{cccccc}
\hline \multirow{2}{*}{ methods } & \multicolumn{2}{c}{ final position errors } & \multicolumn{3}{c}{ final attitude errors $\left({ }^{\circ}\right)$} \\
\cline { 2 - 6 } & $\begin{array}{c}\text { absolute } \\
\text { value }(\mathrm{m})\end{array}$ & $\begin{array}{c}\text { percentage } \\
(\%)\end{array}$ & pitch & roll & yaw \\
\hline INS & 59906 & 1450.9 & 1.935 & -0.946 & 0.181 \\
VNS & 66.706 & 1.615 & 6.075 & 12.176 & 17.681 \\
INT1 & 18.845 & 0.456 & 1.649 & -1.464 & 0.810 \\
INT2 & 5.908 & 0.143 & -1.381 & 0.648 & -0.105 \\
\hline
\end{tabular}

\section{CONCLUSION}

The traditional INS/VNS models which utilize the relative motion difference between INS and VNS, make an approximation between definitions of the inertial measured attitude error and the misalignment angle. Analysis under typical conditions presents that the norm of the approximation error can exceed the norm of misalignment angle, which cannot be ignored. An improved measurement model is presented, which defines the same inertial measured attitude error as the misalignment angle to avoid the approximation. In the lunar-based simulation, the position accuracy of the method with proposed model is improved by $87.07 \%$ compared with that of the traditional model. In the earth-based experiment, the position accuracy is improved by $68.64 \%$. In summary, the simulation and experiment both demonstrate that the method used the proposed model can achieve higher accuracy of positions and attitudes than that used the traditional model.

\section{ACKNOWLEDGMENT}

The research presented in this paper has been supported by the National Natural Science Foundation of China (61233005), the Program for New Century Excellent Talents in University (NCET-11-0771), and the grant of China Scholarship Council (CSC 201303070248). The authors wish to express their gratitude to colleagues for their selfless assistance and valuable comments.

\section{REFERENCES}

[1] Liu Chuankai, Wang Baofeng, Wang Jia, et al. Integrated INSand vision based orientation determination and positioning of CE 3 lunar rover [J]. Journal of Spacecraft TT\&C Technology, 2014, 33(3) : 250-257.

[2] Maimone M, Cheng Y, Matthies L. Two years of visual odometry on the mars exploration rovers [J]. Journal of Field Robotics, 2007, 24(3): 169-186.

[3] Li M, Mourikis A I. Optimization-based estimator design for vision-aided inertial navigation [J]. Robotics, 2013, 241-248.

[4] Tardif J-P, George M, Laverne M, et al. A new approach to vision-aided inertial navigation [C]. proceedings of the IEEE/RS International Conference on Intelligent Robots and Systems. Taipei, Taiwan:IEEE,2010,10: 4161-4168.

[5] Mourikis A I, Roumeliotis S I. On the treatment of relative-pose measurements for mobile robot localization [C]. proceedings of 
IEEE International Conference on Robotics and Automation, IEEE, 2006: 2277-2284

[6] Roumeliotis S I, Johnson A E, Montgomery J F. Augmenting Inertial Navigation with Image-Based Motion Estimation [M]. International Conference on Robotics and Automation. 2002: 4326-4333.

[7] Fang Q, Huang S X. UKF for Integrated Vision and Inertial Sensors Based on Three-View Geometry [J]. Sensors Journal, IEEE, 2013, 13(7): 2711-2719.

[8] Titterton D H, Weston J L. Strapdown Inertial Navigation Technology (2nd Edition) [M]. UK: Institution of Engineering and Technology, 2004.

[9] Lowe D G. Distinctive Image Features from Scale-Invariant Keypoints [J]. International Journal of Computer Vision, 2004, NO.2): 91-110.
[10] Lu W, Xiang Z, Liu J. High-performance visual odometry with two-stage local binocular BA and GPU [C]. proceedings of the Intelligent Vehicles Symposium (IV), Australia: IEEE, 2013,7: 1107-1112

[11] Wang Baofeng, Zhou Jianliang, Tang Geshi, et al. Research on visual localization method of lunar rover [J]. Science China., 2014 44(4):452-60. in Chinese

[12] Olson C F, Matthies L H, Schoppers M, et al. Rover navigation using stereo ego-motion [J]. Robotics and Autonomous Systems, 2003, 43(4): 215-229

[13] Geiger A, Lenz P, Stiller C, et al. Vision meets robotics: The KITTI dataset [J]. International Journal of Robotics Research (IJRR), 2013, No.11): 1231-1237. 